e-Ciencias de la Información

Revista electrónica publicada por la

Escuela de Bibliotecología y Ciencias de la Información, Universidad de Costa Rica, 2060 San José, Costa Rica

e-Ciencias de la Información

Revista electrónica semestral, ISSN-1659-4142

Volumen 4, número 2, miscelánea 2

Julio - Diciembre, 2014

Publicado 1 de julio, 2014

http://revistaebci.ucr.ac.cr/_

\title{
ACCESO ABIERTO Y SOFTWARE LIBRE
}

Manuel Alejandro Echeverría

\section{(c) (1) (2)(2)}

Protegido bajo licencia Creative Commons

Universidad de Costa Rica 


\title{
Acceso Abierto y software libre
}

\author{
Manuel Alejandro Echeverría ${ }^{1}$
}

Fecha de recibido: 09 de diciembre del 2013

Fecha de aceptado: 19 de febrero del 2014

\section{INTRODUCCIÓN}

Con el desarrollo de las TIC (tecnologías de la información) a finales del siglo XX, especialmente el de la Internet, se da inicio a la sociedad de la información, la cual se cimienta en la economía del conocimiento, la gestión, producción y consumo de la información, necesaria para el desarrollo de las sociedades humanas. Es aquí cuando empiezan a tomar fuerza los conceptos de software libre, acceso abierto (open access), el cual incluye los repositorios de libre acceso, y datos abiertos (open data), todo esto para lograr libertad en el conocimiento.

En los últimos años se ha puesto de moda el concepto de software libre, y las premisas de reemplazar las aplicaciones privativas (Windows y Microsoft Office) por las de acceso abierto, empezando por los navegadores web; para lograrlo se requiere de ciertos requisitos como disponer de buenas conexiones a Internet, madurez y adiestramiento por parte de los usuarios, además de que las aplicaciones alternativas logren superar a las privativas.

Primero, se detallará el movimiento acceso abierto (OA), y luego el software libre y aplicaciones abiertas para bibliotecas con las ventajas y desventajas de estas. Seguidamente, se realizará una comparación entre algunas aplicaciones de libre acceso y las privativas correspondientes.

\section{MOVIMIENTO PRO-ACCESO ABIERTO (OA)}

También llamado open access (OA, incluye open data o datos abiertos), el movimiento pro-acceso abierto tiene como propósito el ingreso a las investigaciones científicas de forma gratuita, es decir, sin tener que pagar a las editoriales, debido a que estas aumentan los precios y en muchos casos dificultan la disponibilidad de las publicaciones. No obstante, por medio de la web se hacen más accesibles, ya que los gastos de distribución y de impresión se reducen. La web "ha reducido las inequidades y revolucionado los flujos de información, las formas de acceso y los intercambios informacionales" (Torricella, Lee y Huerta, 2008, p. 3).

\footnotetext{
${ }^{1}$ Universidad de Costa Rica, Escuela de Bibliotecología y Ciencias de la Información. COSTA RICA. echeverria.ts849@yahoo.es
} 
Los datos abiertos (open data) se pueden definir, según Duran (2012),

como información expuesta en la Web en formato tabular de números, estadística, métricas, datos micro y macro económica geo-referenciada o no, de todo tipo de temas, sin restricciones de propiedad intelectual o mecanismos de control en cuanto acceso; entre los tipos de contenidos de los datos abiertos publicados se pueden encontrar geo data (contenidos geográficos), cultura, ciencias, financiamiento, estadísticas, clima, ambiente y transportes. (p. 181-182)

La información publicada como datos abiertos tiene tres características esenciales:

Ser accesibles preferiblemente vía Internet, están en formato digital, dicho formato permite que sean reutilizables por diversas aplicaciones o sistemas, además de tener un licenciamiento de libre restricción en el uso y distribución; todo esto para ponerlos a disposición del público. (Duran, 2012, p. 181)

Los formatos de los datos abiertos son XLS, XLSX, CSV, DOC, ODF, ODP, PDF, TXT, ODT, entre otros. Los archivos deben estar codificados o transformados con un formato UTF-8.

Se han hecho múltiples declaraciones, las cuales definen y apoyan el movimiento OA, tales como:

* La Declaración de Budapest (1999), primera declaración pública e internacional de acceso a la información científica, la cual favorece la libre circulación de conocimiento.

* La Declaración de Bethesda (2003).

* La Declaración de Berlín (2007).

Aparte de dichas aclaraciones, países como Estados Unidos (con el gobierno de Obama), Inglaterra, España, Chile, Uruguay, Brasil, Colombia, entre otros más, promueven los datos abiertos.

\section{En Costa Rica,}

veinte instituciones ya aplican los datos abiertos (municipalidades, Instituto Nacional de Estadística y Censos, CCSS, las universidades estatales, Defensoría de los Habitantes, Poder Judicial, Gobierno Digital de Costa Rica y ministerios) donde se han publicado un total de 1075 de archivos Open Data hasta Febrero del 2013. (Duran, 2012, p. 181)

Open access propone dos estrategias con recursos electrónicos vía web:

> Revistas OA: sin costo alguno, muchas veces arbitradas, cuyos editores garantizan la preservación e integridad de los contenidos; están orientadas a ganar adeptos entre los autores, además de incentivarlos para publicar en estas revistas.

- Repositorios OA: esta estrategia permite el acceso gratuito a la producción bajo el control (copyright) de las editoriales; en estos se colocan los preprints (documentos que carecen de control de calidad) y postprints (tienen control de calidad editorial) aportados por revistas que apoyan el OA. Según Soares y Horsth (2012, p. 36) promueven: 
$\checkmark$ Acceso libre para texto completo de resultados de investigación.

$\checkmark$ Diseminación y promoción de la investigación.

$\checkmark$ Preservación a largo plazo de los resultados de la investigación.

Apoyo para evaluación administrativa.

Los requisitos para la creación y el mantenimiento de repositorios y revistas de acceso abierto según Torricella, Lee y Huerta (2008, p. 4) son los siguientes:

a) Compromiso oficial de la institución o de la editorial, la cual coloca su producción en OA, para publicar su producción científica en Internet gratuitamente y mantenerla en línea por tiempo indefinido.

b) La institución o editorial debe incluirse en el registro de repositorios de libre acceso (ROAR, por sus siglas en inglés); al incorporase a este se hace público el compromiso de proporcionar acceso abierto a los resultados de sus investigaciones.

c) Los archivos digitales de las instituciones y los sitios de las revistas deben cumplir con el protocolo de iniciativa de archivos abiertos para la recolección de metadatos (OAI-PMH, por sus siglas en inglés); este permite a los buscadores especializados rastrear los repositorios OA y su recuperación.

Los repositorios y el acceso abierto son esenciales para la consolidación de las bibliotecas virtuales (muchas veces llamadas bibliotecas digitales) en la web; además, han permitido la consolidación de comunidades de usuarios con el fin de promover y compartir toda clase de conocimiento. Quizás donde más se han creado repositorios han sido en el software libre (especialmente en Costa Rica, más adelante se hablará de esto).

Algunos repositorios muy conocidos son Pubmed Central (PMC), CiteSeerx, arXiv, Kerwa y Latindex.

\subsection{Tipos de Repositorios}

Repositorios institucionales (RI): colección digital conformada únicamente por la institución a la cual sirve y solo es accesible para los funcionarios de esta.

Repositorios de investigación: son apoyados por órganos financiadores que buscan capturar el conjunto de resultados de una investigación.

Repositorios nacionales: se orientan a promover e incentivar la producción académica.

Repositorios temáticos (RT): están constituidos por una colección de objetos digitales producto de investigaciones cuyo objeto de estudio es un campo disciplinario determinado; aceptan colaboraciones de diferentes instituciones y tienen sus áreas de estudio clasificadas. Son una buena estrategia para la publicación de áreas emergentes, además promueven la divulgación del conocimiento. Este tipo de repositorio es el más utilizado. 


\section{SOFTWARE LIBRE}

Iniciado en 1983 por Richard Stallman con el objetivo de crear un sistema operativo compatible con Unix el cual fuera de código abierto donde cualquier persona pudiese utilizarlo, modificarlo y adaptarlo a sus necesidades. En 1985 Stallman funda la Free Software Foundation (FSP) con la función de promover el software libre. En 1989 la FSP publica la primera versión de GNU (General Public License). (Chinchilla, 2011, p. 2-3)

En la práctica, existen dos corrientes: open source software (software de código de fuente abierto) y free software (software libre); estos coinciden en muchos aspectos y por lo general se complementan en variados proyectos.

Pero la diferencia está en que el software libre no es del todo gratuito (en inglés Software Libre se escribe Free Software, y la palabra free se usa tanto para indicar libre como gratis), aunque no se paga por la licencia del producto, pero si es necesario pagar por el servicio de desarrollo, de instalación o de capacitación, además de un CD o DVD para grabar la aplicación y en muchas ocasiones es comercial, por ejemplo Red Hat Linux Enterprise Server , a diferencia del OA que siempre va a ser abierto. (Soares y Horsth, 2008, p. 5)

El objetivo de esto es brindar libertades a los usuarios, donde el acceso al código fuente es un requisito. Estas libertades son:

$>$ La libertad de usar el programa con cualquier propósito (libertad 0).

$>$ La libertad de estudiar cómo funciona el programa y adaptarlo a sus necesidades (libertad 1).

$>$ La libertad de distribuir copias, con lo que se puede ayudar a otros colegas (libertad 2).

$>$ La libertad de mejorar el programa y publicar las mejoras de modo que toda la comunidad se beneficie (libertad 3).

\section{APLICACIONES DE SOFTWARE LIBRE PARA BIBLIOTECAS}

La automatización para bibliotecas inició en la década de 1960, donde el primer gran aporte fue el formato MARC (Machine Readable Cataloging), aún vigente hasta nuestros días, creado por la Biblioteca del Congreso.

En los 1970s la Organización Internacional del Trabajo con el objetivo de agilizar los procesos bibliotecarios desarrolló el Integrated Set of Information System (ISIS), el cual se distribuyó globalmente, en los 1980s partiendo de este, la UNESCO se desarrolló el MicroISIS para MS-DOS, años más se implementaría para sistema operativo Windows bajo el nombre WinISIS. (Herrera, 2012, p. 3-4)

Herrera (2012) nos enseña, a través de la tabla 1, algunas aplicaciones de gran utilidad para bibliotecas.

En la actualidad existe una amplia gama de aplicaciones de código abierto para unidades de información; estas se dividen en tres categorías, las cuales van desde lo más sencillo, que es 
la automatización del catálogo, hasta sistemas integrales de gestión de bibliotecas (SIGB). Según Chinchilla y Fernández (2012, pp. 5-15), se clasifican en:

A. Primer nivel: automatización del catálogo. Software especializado en control bibliográfico y en el módulo de catalogación; algunos ejemplos son WinISIS, ISISMar, Catalis y Biblioteca PHP.

B. Segundo nivel: repositorios de información. Mejor conocidos como bibliotecas virtuales. Estas constan de una base de datos y una interfaz de búsqueda web para la recuperación de archivos digitales; algunos ejemplos son E-Prints, Dspace, Greentone, Resource Space, Google Custom Search, iAH.

C. Tercer Nivel: automatización integral. Estos son los SIGB, que incluyen todos los módulos (circulación, préstamo, adquisición, catalogación), catálogo en línea, generador de reportes estadísticos, formatos internacionales como el MARC21 y Dublin Core. Existen tres niveles: bibliotecas pequeñas, medianas y grandes. Los niveles siguen seis variables referentes a características de la unidad de información, tales como: tipo de unidad de información, tamaño de colección, tipos de materiales, cantidad y tipo de usuarios, recursos humanos y capacidad técnica y económica. Algunos ejemplos de estos softwares integrales son Koha, OpenBiblio, Open MarcoPolo, PHPmyBibli, EspaBiblio, Biblio, EMILDA, GNUTecay y WebLis.

\section{VENTAJAS Y DESVENTAJAS DE LAS APLICACIONES DE SOFTWARE LIBRE}

\subsection{Ventajas del software libre}

Entre las ventajas del software libre se encuentran:

* El acceso al código permite que cualquier usuario pueda realizar mejoras al producto (parametrizar según las necesidades del cliente o de la empresa); esto es muy necesario en las grandes empresas, que requieren el empoderamiento del código fuente, lo cual los hace más flexibles y ágiles para el uso de súper computadoras empresariales, servidores y grandes bases de datos, cosa que el software privativo (Windows Server) no permite.

* Ahorro de recursos monetarios, ya que no requiere compra de licencias.

* La posibilidad de encontrar soluciones para cada nivel de automatización de bibliotecas en el cual se desea involucrar a la organización (automatización de catálogos, bibliotecas virtuales o automatización integral).

* El software libre y los GNU gozan de grandes comunidades de usuario, las cuales intercambian experiencias, comunican sus experiencias y dan soluciones conjuntas.

* Muchas aplicaciones de software libre, como los sistemas operativos y los reproductores multimedia, son más livianas (consumen menos memoria RAM y menos recursos del computador) que las aplicaciones de sistemas privativos (Windows, Cyberlink PowerDvd, etcétera).

* En cuanto a la instalación de un software de gestión integral para bibliotecas, este solo se instala en el servidor y se accede por medio de los navegadores web en 
contraste con las versiones privativas, que se deben instalar en el servidor y en todas las computadoras de usuarios.

* Los sistemas operativos de software libre basados en Linux y sus derivaciones no requieren de antivirus, debido a que se les corrigen las pulgas y errores constantemente y de forma eficiente.

La tabla 2 muestra la comparación de costos de licencias de aplicaciones de pago versus aplicaciones libres.

Además, se puede afirmar que existe una preferencia y una mayor facilidad de manejo de los navegadores web gratuitos y alternativos (Firefox, Chrome y Opera) en contraste del Internet Explorer (considerado por muchos el patito feo de Microsoft), por las siguientes premisas:

$\checkmark$ Son multiplataforma, están disponibles para Windows, Macintosh, Linux, Android, i-OS y Windows Phone.

$\checkmark$ Se actualizan constantemente, por lo general cada dos meses, a diferencia del IExplorer que lo hace una vez al año, dos años o más.

$\checkmark$ Son muy flexibles, ligeros y en algunos casos más innovadores (el navegador web Opera) que las versiones de privativas (I-Explorer y Safari).

$\checkmark$ Instalación rápida y fácil (en contraste con las últimas versiones del Internet Explorer, en las cuales se debe reiniciar el sistema al momento de realizar una actualización y en ciertas ocasiones retardan la pc. Además, una versión de IExplorer se crea solamente para correr en dos o tres versiones de Windows, por lo cual una versión de Windows dispondrá de no más de tres versiones (cuatro, en el caso de Windows 7) de dicho navegador.

$\checkmark$ Disponen de una amplia gama de complementos (extensiones) y temas (cambio de apariencia), los cuales se pueden quitar cuando el usuario lo desee (en contraste con el I-Explorer que al momento de deshabilitar las extensiones, y sobre todo las barras herramientas, no se pueden quitar a menos que se desinstalen desde el panel de control, donde muchas veces se requiere de permisos de administrador).

$\checkmark$ Pueden ofrecer soporte por tiempo adicional (tal fue el caso de Mozilla Firefox y Opera con Windows 2000, el cual caducó en 2010, pero los fabricantes de dichos navegadores le dieron soporte por dos años más, mientras que la última versión de Internet Explorer para el Win2000 fue la 6 del año 2001); en el caso de Ubuntu, se les da soporte hasta la fecha de caducidad de este.

\subsection{Algunas desventajas del software libre}

Sin embargo, el software libre también presenta desventajas como:

> Algunos sistemas no cuentan con una casa comercial para brindar una guía y soporte técnico al proyecto.

$>$ La instalación y puesta en marcha de un software libre es más compleja, requiere de más esfuerzo y tiempo.

$>$ Muchas aplicaciones frecuentemente requieren de software adicional, el cual es frecuentemente más difícil de instalar (requiere de una serie de comandos en la 
terminal de Ubuntu, y muchas veces no hay asistente de instalación con interfaz gráfica) y administrar que el programa de automatización en sí. Además, deben instalarse los motores de base de datos (como MySQL o el servidor Apache, y programas de desarrollo como Java) y de complementos específicos.

$>$ En muchos casos, el personal bibliotecario no cuenta con una sólida formación en el área de informática para poder implementarlo.

$>$ Requiere de una amplia capacitación para poder adaptar la aplicación, acondicionar el hardware y poder familiarizarse con el programa.

$>$ A pesar de que el software libre es de gran utilidad para las bibliotecas y en aplicaciones educativas, todavía es muy complejo y no goza del suficiente soporte.

Todavía no existe una madurez con el uso de software libre.

En el caso de los sistemas operativos de software libre de Canonical, como Ubuntu (y sus derivados como Xubuntu, Lubuntu, entre otros), pueden ser altamente accesibles para el uso de servidores, bases de datos y, en muchos casos, en equipo de oficina, especialmente al momento de navegar en páginas web maliciosas (ya que Ubuntu tiene una mayor resistencia a virus que Windows y Macintosh), pero no son lo suficientemente genéricos para uso doméstico (hogareño), por las siguientes razones:

$>$ Algunos hardware como los celulares (Nokia con Windows Phone) no son compatibles con Ubuntu, por lo cual se debe realizar un reemplazo de dicho hardware (esto requiere de una inversión adicional) y limitarse a comprar solo ciertas marcas que sí ofrezcan soporte con Ubuntu o, en el peor de los casos, pedir prestado una pc que disponga de Windows o Mac.

$>$ Tienen poca diversidad de aplicaciones (muchas son muy sencillas en comparación con las existentes en Windows, además de que este goza de miles de opciones).

$>$ En el uso de juegos (gamers), aunque tal vez los juegos novedosos ofrecen soporte para Ubuntu, en la mayoría de los casos se tiene que codificar con la aplicación Wine (programa para emular aplicaciones de Windows) para poderlos ejecutar, pero la parametrización del Wine y de los juegos es muy compleja en la mayoría de los casos, y los más viejos son exclusivos para Windows.

$>$ Es un sistema esclavo (muy dependiente) de la Internet, ya que exige la conexión de internet (una velocidad de $2 \mathrm{Mbs}$ en adelante) para poder instalarlo, configurarlo e implementarlo, de lo contrario no se podría. En nuestro país, solo 24,06 de 100 hogares que disponen de por lo menos una pc, tienen acceso a la web (Rectoría de Telecomunicaciones 2011, p. 19); por ello, dicho sistema podría aumentar la brecha digital, a diferencia de Windows que no requiere de conexión a Internet para su instalación y la conexión no es tan necesaria a la hora de instalarlo.

$>$ Sus repositorios de complementos (vía web) y de drivers (controladores) tienen una caducidad programada (su periodo de vida es más corto que el de Windows).

$>$ Es poco amigable porque requiere de muchos comandos por terminal a la hora de realizar configuraciones e instalaciones de aplicaciones, a diferencia de Windows, que realiza las configuraciones con un asistente de instalación con interfaz gráfica. 
Esto afecta el adiestramiento de usuarios novatos y de personas de edad madura que dan sus primeros pasos en el aprendizaje de la informática; es un sistema para usuarios avanzados.

$>$ El explorador de carpetas es sencillo, las opciones de vista son pocas, la opción de detalles de los archivos es escasa, el panel de navegación no es expandible.

$>$ Muchas de las opciones de efectos, extras y transiciones, las cuales vienen por defecto en Windows, en Ubuntu son escasas y requieren de una posterior instalación de complementos vía web.

$>$ Requiere de muchos ajustes y configuraciones por comandos en la terminal (especialmente a la hora de conectar celulares para que el sistema los pueda reconocer); en la mayoría de los casos se requiere de conexión a Internet para descargar complementos.

Existen diversas aplicaciones libres como VLC (reproducción de video y películas), Mozilla Firefox y Thunderbird (bandeja de correo electrónico), Audicity (grabación sonido), Calibre (gestión y edición de epubs) y Cmap Tools (para la creación de mapas conceptuales), las cuales gozan de buena aceptación por los usuarios. No obstante, existe polémica en el reemplazo de Microsoft por LibreOffice debido a las siguientes razones:

MS-Office tiene más de veinte años de experiencia (su primera versión como suite apareció en 1992, pero sus aplicaciones iniciaron en la década de 1980) en contraste con LibreOffice, el cual apareció a finales del 2010, originado del OpenOffice (este empezó en el 2001; tiene doce años de existencia).

* LibreOffice no abre ni lee los archivos creados por MS Office de manera óptima; tiene dificultades para reconocer las imágenes y formas insertadas (triángulos flechas, cuadros, entre otras), tablas, y en muchos casos desconfigura los textos, márgenes, tablas y diapositivas creadas por el MS Office.

* Microsoft Office, en sus más de viente años, ha estado en la vanguardia de la innovación (su primera aplicación fue Word en 1983, su primera versión fue para DOS, pero esta ofrecía soporte para el uso de Mouse, y actualmente ofrece soporte en la Nube), ha hecho cambios de interfaz (dejó de usar la tradicional barra de herramientas y la cambió por la barra de cinta en sus tres últimas versiones, además del botón archivo, el cual muestra datos propiedades de los documentos) y de íconos es su travesía, mientras que LibreOffice ha sido muy estático y apegado a la tradicionales barras de herramientas (las cuales se vuelven insuficientes en monitores angostos, inferiores a resoluciones de 1152 x 768 pixeles), que pueden ser algo obsoletas.

* LibreOffice consume más memoria RAM que MS Office y, en ciertos casos, dura más segundos para poderse ejecutar; los usuarios con máquinas de pocos recursos se ven muy afectados.

* MS Office goza de más soporte de instituciones de enseñanza, como escuelas, colegios, INA, AJECO y la Cámara de Industrias de Costa Rica, las cuales dan cursos de capacitación que demandan el uso de Microsoft Office. 


\section{CONCLUSIONES}

Con este trabajo, se puede determinar la importancia del libre acceso para compartir la información por medio del uso de herramientas y aplicaciones digitales gratuitas. Es necesario seguir y apoyar el movimiento de la información libre gratuita y al servicio de la sociedad. En este caso, el acceso abierto ha permitido la creación de blogs, repositorios y comunidades, las cuales comparten y aportan información de suma utilidad.

La importancia del software libre (con código abierto para todo usuario) es fundamental a la hora de implementar aplicaciones útiles para bibliotecas. Además, son un gran ahorro monetario para las instituciones, siempre y cuando se tenga personal capacitado para implementarlo. Las bibliotecas utilizan software de automatización en tres niveles, y para cada nivel existe una amplia gama de aplicaciones gratuitas, las cuales deben ser tomadas en cuenta con cuidado y estudio.

Existe una gran aceptación de los navegadores libres, por ser más flexibles en contraposición a los privativos. Sin embargo, todavía no hay madurez en cuanto al uso del software libre respecto a la implementación del sistema operativo Ubuntu, especialmente en el uso doméstico. Esto se debe en parte por una insuficiente disponibilidad de conexión de Internet en los hogares, la falta de soporte de fabricantes de software y hardware, y la falta de madurez de los usuarios; además, dicho sistema requiere de un exceso de configuraciones para funcionar que el Windows no necesita, o bien, ya trae por defecto.

Asimismo, existe un contraste entre el uso y aceptación del Microsoft Office y LibreOffice, ya que el segundo es poco innovador y apegado a lo tradicional, mientras que el primero es muy innovador, con una larga experiencia, pero es pagado. 
e-Ciencias de la Información • Volumen 4, número 2, miscelánea 1, Jun - Dic, 2014

\section{REFERENCIAS}

Amazon. (2013a). Adobe photoshop extended cs6 student and teacher edition [download] [legacy version]. Recuperado de http://www.amazon.com/Adobe-PhotoshopExtended-StudentDownload/dp/B007USGD60/ref=sr_1_3?ie=UTF8\&qid=1385095048\&sr=8$3 \&$ keywords $=$ adobe + photoshop $+\mathrm{cs} 6$

Amazon. (2013b). Microsoft windows server 2012 standard - 2 cpus/2vm base license. Recuperado de http://www.amazon.com/Microsoft-Windows-Server-2012Standard/dp/B0093CB30M/ref=sr_1_5?ie=UTF8\&qid=1385096524\&sr=8$5 \&$ keywords $=$ windows + server +2012

Amazon. (2013c). Nod32 antivirus version 6 - 3 users. Recuperado de http://www.amazon.com/ESET-EAVH-N1-3-1-RBX-NOD32-AntivirusVersion/dp/B00AJXEP2C/ref=sr_1_1?ie=UTF8\&qid=1385096383\&sr=8$1 \&$ keywords=eset+nod32+antivirus +6

Calle, N. (2011). Sistemas operativos. [Presentación de diapositivas]. Recuperado de http://www.slideshare.net/narcizacalle/windows-linux-mac-8485715

Chinchilla, R. (2011). El software libre: una alternativa para automatizar unidades de información. Revista Bibliotecas, 29 (1). Recuperado de http://www.revistas.una.ac.cr/index.php/bibliotecas/article/view/1557

Chinchilla, R. y Fernández, M. (2012). Bibliotecas automatizadas con software libre: establecimiento de niveles de automatización y clasificación de las aplicaciones. Revista Bibliotecas, $\quad 30 \quad$ (2). Recuperado de http://www.revistas.una.ac.cr/index.php/bibliotecas/article/view/4912

Duran, O. (2012). Informe anual hacia la sociedad de la información y el conocimiento 2012. San José, Costa Rica: PROSIC. Recuperado de http://www.prosic.ucr.ac.cr/materiales/informes/informe-2012

Herrera, R. (2012). Implementación de aplicaciones informáticas basadas en software libre en bibliotecas y unidades de información. Revista E-Ciencias de la Información, 2(1). Recuperado de http://revistas.ucr.ac.cr/index.php/eciencias/article/view/1215

Rectoría de Telecomunicaciones. Ministerio de Ciencia, Tecnología y Telecomunicaciones. (2011). Índice de brecha digital en Costa Rica. Recuperado de http://www.telecom.go.cr/index.php/publicaciones/telecom/publicaciones/indice-debrecha-digital-costa-rica/detail

Soares, M. C., Da Silva, C. H. y Horsth, I. (2012). Los repositorios temáticos en la estrategia de la iniciativa Open Access. Nutrición Hospitalaria, 27(2), 34-40. Recuperado de http://scielo.isciii.es/scielo.php?pid=S0212$16112012000800005 \&$ script $=$ sci_arttext

Torricella, R. G., Tenorio, F, y Huerta, V. (2008). Acceso abierto y software libre: premisas para la independencia tecnológica. ACIMED, 17(2), 33-44. Recuperado de http://dialnet.unirioja.es/servlet/articulo?codigo $=2594963$ 


\section{TABLAS, CUADROS Y FIGURAS}

\section{Tabla 1}

Aplicaciones de Software Libre que se pueden usar en una biblioteca o unidad de información.

\begin{tabular}{|l|l|}
\hline \multicolumn{2}{|c|}{ Aplicaciones Libres y Sus Funciones } \\
\hline Aplicación & Objetivo \\
\hline Linux Ubuntu & Sistema operativo \\
\hline LibreOffice & Suite de ofimática \\
\hline Open Biblio & Sistema de gestión de bibliotecas, (incluye OPAC) \\
\hline Koha & Sistema de gestión de bibliotecas, (incluye OPAC) \\
\hline Tema Tres & Tesauro en línea \\
\hline GNU Cash & Contabilidad \\
\hline Dspace & $\begin{array}{l}\text { Repositorio documental en línea con capacidades de } \\
\text { búsqueda mediante descriptores }\end{array}$ \\
\hline Drupal & Gestión de contenidos web basada en módulos \\
\hline Scribus & Programa para maquetación de publicaciones \\
\hline LimeSurvey & Encuestas en línea \\
\hline Open Project & Gestor de proyectos \\
\hline Gimp & Manejo de imágenes \\
\hline InkScape & Dibujo vectorial \\
\hline
\end{tabular}

Fuente: Herrera, 2012, p. 14.

Tabla 2

Precios de las licencias de aplicaciones de pago y libres

\begin{tabular}{|l|l|l|l|l|}
\hline $\begin{array}{l}\text { Tipo de } \\
\text { aplicación }\end{array}$ & Privativa & Costo & Libre & Costo \\
\hline $\begin{array}{l}\text { Software para } \\
\text { gestión } \\
\text { bibliotecaria }\end{array}$ & JANIUM & $\$ 20.000,00$ & KOHA & $\$ 0,00$ \\
\hline $\begin{array}{l}\text { Manejo de } \\
\text { imágenes }\end{array}$ & $\begin{array}{l}\text { Adobe Photo Shop } \\
\text { Extended CS6 Student } \\
\text { and Teacher Edition } *\end{array}$ & $\$ 349.00$ & Gimp & $\$ 0,00$ \\
\hline Antivirus & $\begin{array}{l}\text { Eset Nod32 Antivirus 6 } \\
\text { (3pcs) }\end{array}$ & $\$ 34,90$ & $\begin{array}{l}\text { Si se usa Linux } \\
\text { no se requiere }\end{array}$ & $\$ 0,00$ \\
\hline $\begin{array}{l}\text { Sistema } \\
\text { operativo para } \\
\text { servidor }\end{array}$ & $\begin{array}{l}\text { Windows Server 2012 } \\
\text { Standard (2 cpus) } *\end{array}$ & $\$ 672,73$ & Ubuntu Server & $\$ 0,00$ \\
\hline Fun: Amazn & (2013a, 2013b, 2013c) & & \\
\hline
\end{tabular}

Fuente: Amazon (2013a, 2013b, 2013c). 